\title{
Performance of Pulse Source consisting of an Externally Injected Gain-Switched Laser followed by a Non-linearly/Linearly Chirped Grating in an $80 \mathrm{~Gb} / \mathrm{s}$ OTDM System
}

\author{
R. Maher ${ }^{1}$, P. Anandarajah ${ }^{1}$, A. Clarke ${ }^{1}$, C. Guignard ${ }^{1}$, L. P. Barry ${ }^{1}$, L. Bramerie ${ }^{2}$ and J. Harvey $^{3}$ \\ ${ }^{1}$ RINCE, School of Electronic Eng., Dublin City University, Ireland. ${ }^{2}$ FOTON-ENSSAT / Plate-forme PERSYST, 6 rue de \\ Kérampont, BP 8051822305 Lannion Cedex, France. ${ }^{3}$ Physics Dept. Auckland University, New Zealand.
}

\section{SUMMARY}

Optical Time Division Multiplexing (OTDM) and hybrid OTDM/WDM (Wavelength Division Mulitplexing) technologies are promising ways of increasing the capacity of optical communication systems without increasing the cost (by avoiding high-speed electronics) [1]. One of the key requirements in such highcapacity systems is the development of picosecond optical pulse sources that exhibit excellent temporal and spectral properties. The Temporal Pedestal Suppression Ratio (TPSR), or extinction ratio, is one such vital parameter. In this paper, we characterize the performance of two gain-switched pulse sources, one employing a Nonlinearly Chirped Fibre Bragg Grating (NC FBG) that achieves excellent TPSR ( $>35 \mathrm{~dB})$, and the other using a Linearly Chirped Fibre Bragg Grating (LC FBG) that achieves poor TPSR $(\sim 20 \mathrm{~dB})$, when employed in an $80 \mathrm{~Gb} / \mathrm{s}$ OTDM system. The degraded performance $(3.5 \mathrm{~dB}$ power penalty) in the case of the latter, even though both sources generate pulses that are transform-limited and have durations $<30 \%$ of the $80 \mathrm{~Gb} / \mathrm{s} \mathrm{bit} \mathrm{slot,}$ is attributed to the presence of pulse pedestals. These pedestals cause coherent interactions between individual OTDM channels, thereby resulting in severe intensity fluctuations [2]. Performance verification of 2 ps pulses at a repetition rate of $10 \mathrm{GHz}$, from a commercially available Tunable Mode-Locked Laser (TMLL) pulse source, employed in the same $80 \mathrm{~Gb} / \mathrm{s}$ test-bed is also carried out. This result acts as a reference when encompassed with the overall evaluation.

There are numerous methods available to generate ps optical pulses [3]. One of the simplest and most reliable techniques to generate pulses with low jitter and high Side Mode Suppression Ratio (SMSR) involves externally injecting a gain-switched laser [4]. However, the direct modulation of the laser causes a time varying carrier density in the active region, which results in a frequency chirp across the pulse. This chirp could be used to compress the pulses, using Dispersion Compensating Fibre or LC FBGs, to obtain near transform-limited pulses. However, due to the chirp being nonlinear across the wings of the pulse, this compression typically results in pedestals that make them unsuitable for use in practical systems. In previous work [5], we proposed employing a tailor made NC FBG after the gain-switched laser to achieve direct compression thereby obtaining near transform-limited pulses and a TPSR $>35 \mathrm{~dB}$. This excellent TPSR is achieved not only by the fibre grating having a nonlinear group delay profile that is the inverse of that across the gain-switched pulse directly from the laser, but also by ensuring the fibre grating has a specially adapted transfer characteristic as in Figure 1 (a). The NC FBG has a custom designed non-linear reflective profile that optimizes the output pulse spectrum (Gaussian shape at the grating output) thereby further enhancing the temporal quality of the pulses.
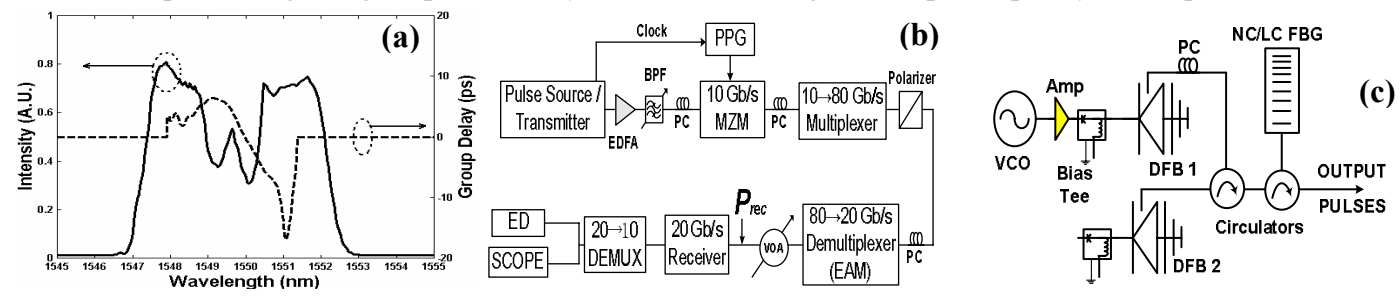

Figure 1: (a) Reflection and group delay profiles of NC FBG (b) $80 \mathrm{~Gb} / \mathrm{s}$ OTDM test-bed (c) Externally injected gainswitched laser with NC FBG \& LC FBG

Figure 1 (b) shows a schematic of the $80 \mathrm{~Gb} / \mathrm{s}$ test-bed used in this work to characterize the performance of three different pulses sources employed in-turn as the transmitter. In the first instance of system performance characterisation, the externally injected gain-switched laser diode was followed by the NC FBG. Secondly, the NC FBG was replaced by the LC FBG. Essentially, as shown in Fig. 1 (c), these two pulse sources consist of a commercially available high-speed $1550 \mathrm{~nm}$ DFB (1) laser that is gain-switched at a repetition rate of $10 \mathrm{GHz}$. External injection, from a second DFB (2) laser operating in CW mode, is carried out to improve the SMSR and the temporal jitter of the gain-switched laser via a circulator. The temporal jitter is measured to be $<1 \mathrm{ps}$ and the SMSR is improved from 5-30 dB. The generated pulses are then spectrally shaped and temporally compressed by the specially fabricated gratings, via a second circulator. The final transmitter consisted of the commercially available TMLL. In all three cases, a Pseudo Random Bit Sequence (PRBS) of length $2^{7}-1$ from a Pulse Pattern Generator (PPG) was used to modulate the $10 \mathrm{GHz}$ pulse train with the aid of a Mach-Zehnder modulator. The resultant STM 64 RZ optical signal is then passed into a passive fibre based interleaver and multiplexed up to 80 $\mathrm{Gb} / \mathrm{s}$. A polarization controller at the input and a polarizer at the output of the multiplexer ensure that the same state of polarization is maintained on each of the tributaries. 
In order to test the performance of the three sources when employed in the $80 \mathrm{~Gb} / \mathrm{s}$ OTDM system, the signal is initially demultiplexed (stage 1) down to $20 \mathrm{~Gb} / \mathrm{s}$ with the aid of an Electro-Absorption Modulator (EAM). The EAM is driven with a $20 \mathrm{GHz}$ sine wave to yield an 8 ps switching window. The signal is then optically pre-amplified prior to being received with the aid of a photodetector after which it is demultiplexed (stage 2) back down to the base rate $(10 \mathrm{~Gb} / \mathrm{s})$ using an electrical demultiplxer. BER measurements are performed for a range of received optical powers (measured before the $20 \mathrm{~Gb} / \mathrm{s}$ receiver stage). The compressed pulses after the NC FBG (bold line) and the LC FBG (faint line) with widths (FWHM) of 3.5 ps and 3.6 ps respectively are shown in Figure 2 (a) (as characterised using Frequency Resolved Optical Gating). The same figure also shows the pulses generated by the TMLL with exhibited widths of 2 ps (FWHM). The associated time bandwidth products of the three pulse sources were $0.45,0.47$ and 0.4 respectively. As can be seen, the pulses compressed by the LC FBG portray a TPSR of about $20 \mathrm{~dB}$ while that compressed by the NC FBG and the TMLL exhibit a TPSR $>35 \mathrm{~dB}$. Figure 2 (b) displays the BER versus received power curves for one of the demultiplexed channels. It can be observed that to achieve a BER of $10^{-9}$ a power penalty of $3.5 \mathrm{~dB}$ is incurred in the case of the LC FBG when compared to the NC FBG. This degraded performance is due to the presence of the pedestals about $20 \mathrm{~dB}$ below the peak of the pulse. These pedestals deteriorate the extinction between the adjacent timeslots of the temporally multiplexed signal thereby leading to intensity fluctuations that causes the BER degradation. A difference of $0.4 \mathrm{~dB}$ was noticed in the case of the commercial TMLL and the optimized gain switched pulse source employing an NC FBG. This variation could be attributed to the difference in pulsewidth $(\Delta \tau \sim 1.5 \mathrm{ps})$, with the narrower pulsewidth leading to a slightly better sensitivity at the receiver.
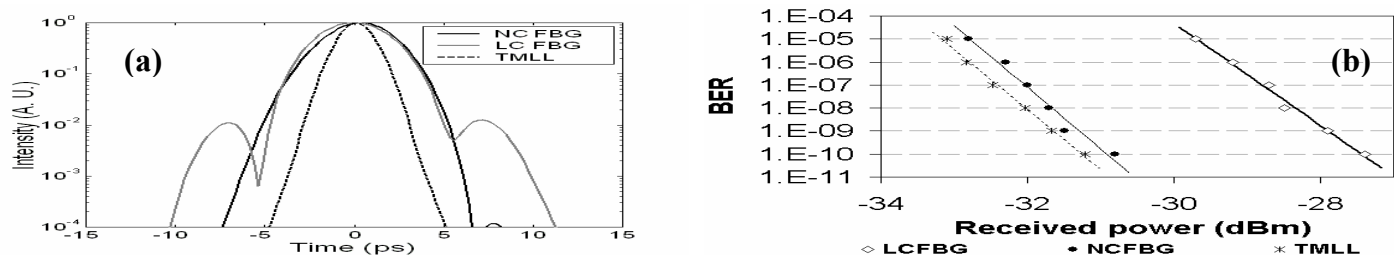

Figure 2: (a) Intensity of externally injected gain-switched pulses after NC FBG (bold), LC FBG (faint) and TMLL (dotted) (b) BER vs received power for NC FBG, LC FBG and TMLL

The system penalties introduced by a poor TPSR and the different pulse widths were characterized by carrying out simulations using Virtual Photonics Incorporated (VPI). The spectral shaping and temporal compression of the gain-switched pulse sources by the NC FBG and the LC FBG were modelled by using a measured optical filter (intensity and group delay parameters obtained experimentally). The pulses from the TMLL were mimicked by using $\operatorname{Sech}^{2}$ pulses that were 2 ps wide. Performance characterization of all 3 pulse sources was carried out by employing them in an $80 \mathrm{~Gb} / \mathrm{s}$ OTDM system. A plot of the BER as a function of received optical power (Fig 3) shows that the system employing gain-switched pulses with NC FBG and LC FBG incur a power penalty of $\sim 0.3$ and $3.5 \mathrm{~dB}\left(@\right.$ BER of $\left.10^{-9}\right)$ respectively with respect to the system that uses the 2 ps $\operatorname{Sech}^{2}$ pulses.

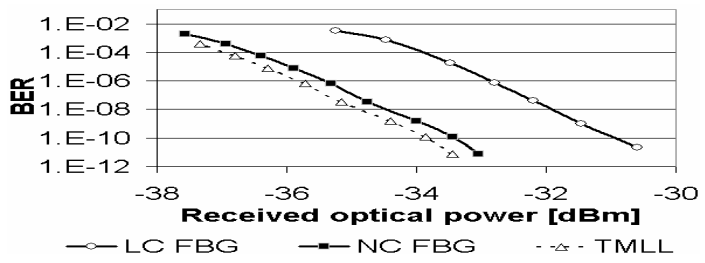

Figure 3: BER vs received power for NC FBG, LC FBG and TMLL

We have demonstrated the excellent performance of an externally injected gain-switched source followed by a NC FBG (nonlinear group delay and nonlinear reflection profile) by employing it in an $80 \mathrm{~Gb} / \mathrm{s}$ OTDM system. A major improvement in system sensitivity $(3.5 \mathrm{~dB})$ achieved by the increased suppression of the temporal pedestals was recorded when compared with a gain-switched source employing a linearly chirped grating. A commercially available mode-locked pulse source, used a benchmark, further stressed the exceptional performance of the proposed pulse source. System simulations show very similar traits and power penalties to the experimental results obtained.

Acknowledgements: This work was supported in part by Enterprise Ireland and Science Foundation Ireland.

\section{References}

1 D. M. Spirit et al, IEEE Commun. Mag., vol. 32, pp 56-62, 1994.

2 M. C. Gross et al, IEEE Photon. Technol. Lett., vol. 14, pp. 690-692, 2002.

3 M. Saruwatari, IEEE J. Sel. Top. Quantum Electron., vol. 6, pp. 1363-1374, 2000.

4 L. P. Barry et al, IEEE Photon. Technol. Lett., vol. 5, pp. 1132-1134, 1993

5 P. M. Anandarajah et al, IEEE J. Sel. Top. Quant. Electron., vol. 12, pp 255-264, 2006. 